\title{
Carnets
}

Revue électronique d'études françaises de l'APEF

Deuxième série - 2 | 2014

Frontières de la chronique

\section{Chronique journalistique et causerie : rapports, formes, enjeux}

\section{Sandrine Carvalhosa}

\section{(2) OpenEdition}

Journals

Édition électronique

URL : http://journals.openedition.org/carnets/1256

DOI : $10.4000 /$ carnets. 1256

ISSN : 1646-7698

\section{Éditeur}

APEF

\section{Référence électronique}

Sandrine Carvalhosa, «Chronique journalistique et causerie : rapports, formes, enjeux », Carnets [En ligne], Deuxième série - 2 | 2014, mis en ligne le 30 novembre 2014, consulté le 19 avril 2019. URL: http://journals.openedition.org/carnets/1256; DOI : 10.4000/carnets.1256

Ce document a été généré automatiquement le 19 avril 2019.

\section{(c) (i) (8)}

Carnets est mis à disposition selon les termes de la licence Creative Commons - Atribution - Pas d'utilisation commerciale 4.0 International. 


\title{
Chronique journalistique et causerie : rapports, formes, enjeux
}

\author{
Sandrine Carvalhosa
}

« [Les chroniqueurs sont] la pluie de sauterelles du Journalisme contemporain. L'Egypte, cette pauvre vieille, maudissait ses sauterelles. Elle les appelait douloureusement une plaie... mais le Journalisme tend son chapeau aux siennes, comme les Croisés, après une sécheresse, tendaient leurs casques à la rosée... Il les recueille, il les ramasse, il les recherche et il les paie des prix fabuleux, qui ne sont pas des fables car il publie, pour qu'on n'en ignore et pour forcer la foi aux choses incroyables, ses traités de Laurent-le-Magnifique avec eux. Importants, ces messieurs? que dis-je ? ils sont indispensables. Ils sont les rois du Journalisme. »

Barbey d'Aurevilly, $1883: 75$

Cette citation de Jules Barbey d'Aurevilly, datée de 1866, est certes peu favorable au genre; elle montre bien cependant la place de choix occupée par la chronique dans la «civilisation du journal» que fut le xix siècle. Pour tenter de cerner le genre de la chronique journalistique, il convient de consulter d'abord les définitions données par les contemporains. Pour le Dictionnaire Universel des Littératures, le mot désigne, « dans le journalisme contemporain, des articles consacrés, sous forme de causerie, d'abord aux faits et aux bruits du jour, puis aux divers sujets de politique, d'histoire et de littérature. » (Vapereau, 1876 : 461) D'après le Grand Dictionnaire Universel du XIX siècle, il s'agit de « (...) certains articles ou feuilletons, écrits au jour le jour, publiés par les journaux et qui sont pour ainsi dire le reflet heure par heure de la vie courante »; ce sont des "productions hâtives, oubliées aussitôt que nées ». Et il précise que «dans ces derniers temps, la chronique s'est glissée dans la plupart des journaux; elle est devenue un besoin pour le lecteur (...) ». A la fin du siècle, de Wogan définit la chronique comme « le commentaire de la nouvelle récente, des bruits de Paris, de ce qui se passe, de ce qui se dit, et le ton 
spirituel et léger de la conversation française est presque le seul que l'on emploie pour écrire ces causeries. » Après avoir donné comme modèles de ce genre, « où il faut surtout la grâce et l'esprit », « MM. Aurélien Scholl, Anatole France, J. Lemaitre, Sarcey, Mirbeau, Caliban ", il ajoute que, pour le "chroniqueur populaire des journaux à un sou, d'autres moyens d'action sont indispensables: intéresser, vulgariser, s'occuper, sans en oublier aucune, de toutes les questions agitées dans l'immense fourmilière, voilà sa tâche. » Pour ce faire, «il aura soin de parler un langage que comprennent bien tous ses lecteurs. » (Wogan, 1899 : 182-183)

2 Ces définitions relèvent, comme éléments constitutifs du genre, un contenu et une forme, dont les frontières paraissent tout de même mouvantes: si l'actualité semble former le contenu des chroniques, elle doit être entendue au sens large, si bien que le champ du chroniqueur n'a pour réelles limites que celles qui lui sont fixées par la ligne éditoriale du journal et par les contraintes législatives; si le ton de la causerie est la forme requise pour cette prose journalistique, il doit cependant s'adapter au lectorat, créant ainsi une première distinction entre chronique et chronique populaire. La notion même de causerie suppose une grande plasticité formelle et la liberté, pour le chroniqueur, de mobiliser à sa guise une grande variété de dispositifs textuels.

3 Tout aussi mouvantes sont les représentations de la chronique. L'accusation première, et qui vaut pour tout le journal, est celle d'avoir tué (ou affadi) la conversation ${ }^{1}$. Plus spécifiquement, le succès de la chronique a pour pendant le mépris dont elle fait l'objet chez certains hommes de lettres (qui s'y adonnent pourtant souvent). Cependant, si les représentations romanesques de la presse (chez Balzac, Flaubert ou les Goncourt) sont bien connues, les plaidoyers en faveur de la chronique le sont moins.

Ce sont donc les frontières de la chronique journalistique que nous questionnerons dans cet article, qui se bornera à la période $1836-1885^{2}$. Quels liens la chronique entretient-elle avec la causerie ? Pourquoi la causerie journalistique devient-elle chronique et est-elle perçue comme un symptôme de décadence voire une menace pour les lettres? Quels enjeux sont attachés à l'écriture de la chronique et à la publication en recueil, dans les discours des contemporains? Tels sont les points que nous examinerons successivement.

\section{Petite chronique de la chronique-causerie}

5 Retraçant l'histoire de la chronique journalistique, Marie-Ève Thérenty explique que «dans [l]es années 1830, la chronique émerge simultanément dans l'ensemble des périodiques ", et que ses transformations sont dues à la " rencontre entre deux écritures, la liste de faits d'actualité et l'observation narrativisée des mœurs ». (Thérenty, 2011 : 955) Les chroniques de la chronique ne manquent pas; au sein même de la chronique, il est fréquent que l'hommage aux prédécesseurs installe le nouveau chroniqueur dans ses fonctions (dans le cadre d'un premier article en forme de préface ou de profession de foi), ou remplisse opportunément les colonnes, quand l'actualité fait défaut ${ }^{3}$. Ces généalogies, peu ou prou identiques, remontent souvent jusqu'à Mme de Sévigné, inscrivant la chronique journalistique dans l'histoire de la causerie écrite, et soulignant ainsi la continuité d'un style et d'une fonction (communiquer des nouvelles) au-delà du changement de support : rechercher des antécédents moins dans l'histoire de la presse que dans les Belles-Lettres revient à donner une forme de caution et de dignité littéraire au genre. Ces généalogies recensent les causeurs de presse marquants, ayant contribué au genre : Étienne de Jouy, et surtout Delphine de Girardin (qui a écrit ses Courriers de Paris 
sous le pseudonyme de "vicomte de Launay ») et Eugène Guinot. Au cours d'une conférence consacrée au « Journaliste », Francisque Sarcey déroule l'histoire du genre, et définit par là même le « bon usage » de la chronique :

(...) La chronique n'est pas une invention contemporaine, elle est éternelle en France. Jamais il n'y a eu de siècle sans chroniqueurs, depuis madame de Sévigné jusqu'à ceux de nos jours. Le XVIII ${ }^{\mathrm{e}}$ siècle en a abondé; l'Empire a eu les siens. Dernièrement, étant dans un château, j'y trouvai l'Ermite de la Chaussée-d'Antin qui amusait tant nos grand'mères. Sauf les détails de toilette, les manches à gigots, les tailles courtes et toutes ces choses de mode qui ont disparu, on croirait lire une chronique de notre temps. C'est plein d'esprit. M. de Jouy était très amusant.

Vous avez tous dans la mémoire les Causeries du vicomte de Launay et les chroniques d'Eugène Guinot, où nos pères ont trouvé tant de plaisir.

De nos jours, un des esprits les plus aimables a renouvelé absolument le genre. Jamais on n'a vu plus de bon sens, de bonne humeur, de gaieté vive que n'en montre le Bourgeois de Paris. Quel art de tout dire en ayant l'air de ne rien dire, de conter de façon à vous les faire trouver toutes nouvelles des histoires que tout le monde connaît! Lisez son récit d'une nuit de baccarat, c'est un chef-d'œuvre, qui restera un chef-d'œuvre pendant trente ou quarante ans.

La chronique ainsi faite, spirituelle et honnête, est un genre excellent. (Sarcey, $1869 \mathrm{~b}: 333)$.

La chronique récupère les moyens et les fonctions de la causerie française, dont elle se veut une incarnation moderne (Diaz, 2011). L'« art tout français » de causer, qui consiste, d'après Sarcey, à « présenter les idées générales sous une forme personnelle, particulière au causeur » trouve sa place désormais dans le journal : « maintenant que la causerie est un peu passée de mode, c'est là qu'on la retrouve. » (Sarcey, 1869a : 20) Dans la nécrologie qu'il consacre à Mme de Girardin le 8 juillet 1855 dans le Figaro, B. Jouvin estime qu'« à travers ce qui semblait, à la majorité des lecteurs, n'être qu'un bavardage amusant et spirituel, circulait la sève gauloise et pétillait le sel français. » Bien plus : «c 'était comme un réveil inattendu de la langue nationale, longtemps humiliée et enrôlée de force, par le mouvement romantique, à la suite des muses de l'art étranger, Shakspeare, Goëthe et lord Byron [sic]. » Mme de Girardin n'a pas seulement « ressuscité (...) les grâces de style, les adorables fantaisies d'imagination » de Mme de Sévigné, mais elle y a encore ajouté « une couche d'observation et de philosophie plus profonde ». La chronique est un genre où se réfléchit le contemporain : il est tout à la fois le miroir de l'actualité et son commentaire spirituel.

7 Avec Le Petit Journal, quotidien à un sou créé en 1863, et son chroniqueur-vedette, Timothée Trimm (Léo Lespès), «chroniqueur ordinaire de Sa Majesté Tout-le-Monde » selon l'expression de Jules Vallès, la chronique se donne une mission d'instruction des masses, par une causerie qui se veut familière. Trimm n'est pas le premier à revendiquer le modèle de la causerie familière (Dumas en particulier l'avait déjà mis en pratique dans sa "Causerie avec mes lecteurs »), mais il invente une poétique singulière de la chronique populaire, qui sera tout à la fois critiquée et reprise par ses concurrents. Les principes d'écriture de cette chronique ont déjà été bien décrits (Thérenty, 2011: 958-962; Chabrier, 2013) : dans ce quotidien apolitique, le chroniqueur réalise la prouesse de livrer, en première page, la causerie de chaque jour. Tout est mis en œuvre pour faciliter la lisibilité de cette écriture-chronique destinée au plus grand nombre: Trimm, en particulier, tronçonne sa prose par des alinéas fréquents qui «forçai[ent] à lire selon la ponctuation; le peuple, obligé de s'arrêter à chaque virgule, où l'on changeait la ligne, comprenait aussi beaucoup mieux, et tout ce que le chroniqueur voulait souligner restait 
aussi bien plus en relief.» (Wogan, 1899: 185) Quant au contenu de ce "premier Timothée ${ }^{4}$ », Trimm le résume fidèlement dans sa chronique du $1^{\mathrm{er}}$ janvier 1865 :

(...) J'ai essayé, sous la forme la plus familière et la moins aride, de mettre à la portée de tous, l'art, la science, la morale vulgarisée.

L'anecdote elle-même, qui n'est que la partie pittoresque de l'histoire, nous a servi à répandre au loin les véritables connaissances utiles.

(...) J'ai cherché tout d'abord à vous distraire.

Et dans la biographie, dans le conte, dans la légende, dans la bibliographie, dans toutes les matières traitées par ma plume rapide, j'ai fait ressortir l'enseignement civilisateur, l'acte héroïque et recommandable, le citoyen fameux par ses vertus et ses talents, l'invention ingénieuse ou le mérite caché.

\section{Le mal chronique}

Le règne de la chronique-causerie - et ses contestations virulentes - se situe véritablement sous le second Empire. Pour Sarcey, «on dit beaucoup de mal de la chronique et des chroniqueurs, qui prennent depuis quelque temps une plus large place dans les journaux ; en effet, si l'on considère ce qu'a été la chronique dans ces dernières années, il n'y a pas, en général, grand bien à en dire. » (Sarcey, 1869b : 333) Répondant à la fois aux contraintes législatives qui s'exercent sur la presse - contraintes qui délimitent strictement le périmètre des conversations et causent un sérieux manque à écrire aux journaux non cautionnés -, à l'impératif de périodicité, et au goût du public pour la chronique, la causerie devient chronique (pour reprendre un jeu de mot de Larousse) et le genre se fige dans des automatismes ou du prêt-à-écrire, dont la critique fournit d'ailleurs de la matière à écrire aux journaux non politiques... et aux chroniqueurs. Sans nulle prétention à l'exhaustivité, nous distinguerons ici trois types de critiques adressées à la chronique : celles qui pointent les abus et les facilités du genre; celles qui, plus sévères, analysent les maux de la chronique en termes de décadence du journalisme et de la société ; celles enfin qui voient dans la chronique une menace pour le livre et un tremplin en trompe-l'œil pour les hommes de lettres.

Le premier type de critiques vise donc les abus et facilités du genre. Une adresse «À MM. Les Chroniqueurs ", lancée depuis le Figaro le 19 décembre $1858^{5}$ (meilleure école pourtant de cette escrime verbale) s'insurge contre les marronniers du journalisme - la " chronique des quatre saisons ", « qui revient régulièrement au printemps avec les petits pois et en hiver avec les ramoneurs »-, et contre les excès de style, la chronique étant devenue « un steeple-chase de l'esprit où il faut à tout prix surpasser les autres coureurs en extravagances et en saillies forcées." Certains chroniqueurs sont ainsi des «saltimbanques de la phrase»:

Attention! On les voit soulever à grands efforts de bras d'énormes périodes chargées d'épithètes ; porter en équilibre sur le bout de leur nez de longs et creux paradoxes; jouer avec une langue disloquée comme un clown du cirque ; jongler lourdement avec les couteaux à pointe émoussée de la polémique littéraire ; faire en arrière le saut de tremplin de la transition forcée, et recevoir et donner un peu partout des coups de pieds inoffensifs, le tout pour le plus grand amusement de la foule, qui leur jette quelques gros sous.

La chronique populaire a aussi ses maux: les détracteurs pointent la conversation dégradée ${ }^{6}$ - voire dégradante ${ }^{7}$ - du Petit Journal, son « savoir coupé, au jour le jour, dans les pages d'un dictionnaire encyclopédique ${ }^{8}$ ", les bévues de Trimm", sa "stérile fécondité ». "Ouvrir quotidiennement le robinet de la causerie du Petit Journal » (Le 
Tintamarre, 21 mars 1869) est en effet un véritable tour de force qui participe puissamment à la fidélisation du lectorat, car comme le dit Trimm lui-même, « la lecture [de ces lignes rapides et sans prétention] est devenue pour vous une sorte d'habitude » (3 octobre 1867). Or, remarque-t-on, il est impossible d'écrire un article de qualité quotidiennement, " avec la ponctualité d'une poule pondant ses œufs ", selon la formule de Jouvin. Le Tintamarre le dira d'ailleurs à Jules Vallès, engagé en février 1866 par Villemessant pour l'article de tête dans son journal L'Événement (lui-même destiné à concurrencer Le Petit Journal). La chronique de Jules Vallès ayant soulevé des protestations de la part des lecteurs (qui estiment que le chroniqueur a trop souvent la « corde triste »), Vallès s'adresse directement à ses lecteurs et lance un sondage, pour savoir s'il doit continuer à occuper la première page du journal. Le Tintamarre lui répond le 11 mars 1866 :

M. J. Vallès voudra bien nous expliquer à ses moments perdus, quel était le but, l'utilité ou l'attrait pour le public, de son article de dimanche dernier, dans lequel il agitait la grave question de savoir s'il devait continuer à occuper chaque jour la première page de l'Événement. (...)

Eh bien ! puisque $M$. Jules Vallès semble tenir à de sincères avis, nous $\mathrm{y}$ allons $\mathrm{du}$ petit nôtre et lui répondons carrément :

NON !...

Non. M. Jules Vallès, dont le talent et l'originalité ne peuvent être mis un instant en doute, ne doit pas continuer ce travail quotidien de vidage qu'il fait depuis quelque temps.

Non. M. Jules Vallès n'est pas fait pour la littérature à l'aune. Qu'il laisse cette corde aux spécialistes qui tirent à 300,000.

Avec ce stupide système de la ponte forcée, on en arrive à quoi !...

A pondre un bon œuf tous les mois. Et encore !...

11 Tout sujet (de polémique) est bon à prendre pour un article: Trimm, fréquemment interrogé sur sa méthode d'écriture, en fera le sujet d'une causerie malicieuse le 3 octobre 1867.

12 La seconde catégorie de reproches, plus sévère, voit dans les maux (mots) de la chronique l'expression, voire une cause, de la décadence du journalisme et de la société. Constatant que des journaux sous le second Empire avaient adopté un "perpétuel bavardage » comme système de remplissage, Vapereau écrit :

Nous voilà bien loin du temps où le journalisme s'offrait à la bourgeoisie comme un sacerdoce, où la presse était considérée comme un des grands pouvoirs de l'État ! Aussi bien, les journaux sont comme les livres, comme le théâtre, ils répondent forcément à l'esprit, aux goûts et aux besoins d'une époque ; qu'ils les flattent ou les combattent, ils sont autant l'effet que la cause du progrès ou de la décadence morale et intellectuelle de la société : une littérature a toujours le journalisme qu'elle mérite. (Vapereau, $1876: 1115)$

13 Du côté de la presse populaire, Trimm, régulièrement attaqué, se fait l'écho dans ses colonnes des accusations de décadence qui visent son journal et sa chronique (2 février 1866) :

(...) Je me trouvai en face d'autres adversaires qui me firent l'honneur de me prendre pour un chef d'école marchant tout droit à la Décadence.

La Décadence ! remarquez donc le joli mot...

Offenbach fait de la musique qui se retient, qui se chante, qui se danse, que nos

filles et nos femmes répètent sur le piano... Décadence !

Hamon fait sourire sur la toile ces harmonieuses figures grecques auxquelles il

donne pour les animer des têtes adorablement parisiennes. Décadence!

Il se présente une chanteuse qui a le geste grotesque et la voix juste, la pantomime 
bouffonne et la méthode sérieuse, et qui représente ainsi l'antithèse, toujours saisissante pour les masses. Décadence!

Et voici un écrivain, le plus petit entre tous, je vous le concède, qui trouve par hasard le moyen d'intéresser la foule sans parler du Corps législatif et du Sénat, de M. de Bismark et du président Johnson, et vous répétez, ô mes antagonistes ! votre mot favori : Décadence! décadence toujours!

Dans l'article qu'il consacre à "Timothée Trimm et Thérésa", Vallès, prenant le contrepied des accusations de décadence, dit avoir « l'espérance et point la honte » face au succès de ces deux figures populaires, signes d'une rénovation en marche :

Qui parle du goût qu'on insulte et de la morale qu'on compromet? Ce n'est pas à son aurore qu'il faut demander à une littérature nouvelle, la mesure et la grâce. Nous sommes à l'époque de transition. Qu'importent quelques fautes par ci, quelques cris rauques par là ? C'est la nécessité d'aujourd'hui, ce n'est pas le Code artistique de demain. (Vallès, 1866)

Troisième type d'attaques : celles qui ont pour objet les rapports de la chronique et de la littérature. La chronique, effroyable minotaure qui exige une écriture régulière et importante, «vide » les hommes de lettres et dévore le temps nécessaire à l'écriture du livre. Élargissant son propos aux différentes rubriques du journal, Vapereau stigmatise le « nouveau genre de littérature » que forment les recueils d'articles, « composés de riens vieillis et décolorés » :

Le journal tend de plus en plus à supplanter le livre. Il appelle à lui tous les écrivains de valeur ou de quelque renom; il met en réquisition tous les talents, les éparpille, les gaspille en menue monnaie. Il dévore par miettes et parcelles ce temps précieux que réclament les œuvres importantes. Le livre s'en va. Cependant le volume reste : on le compose avec ces fragments tombés au jour le jour d'une plume affairée. (Vapereau, $1866: 239$ )

Pourtant, concède-t-il, « l'esprit qui surnage toujours et peut soutenir les choses les plus éphémères, donne à quelques-uns de ces recueils une apparence de vie ", et il reconnaît un vrai talent à des chroniqueurs tels que Noriac ou About. Pour ses contempteurs, la chronique est un miroir aux alouettes; les contrats mirifiques de quelques « maréchaux de la chronique ", leur gloire (Trimm a un champagne à son nom, publie avec succès ses causeries en recueil, et se retrouve à l'affiche de conférences littéraires) ne doivent pas faire illusion : la chronique prend bien plus aux chroniqueurs qu'elle ne leur donne. Les chroniqueurs vivent dans un régime de dépense perpétuelle : dépense d'argent, dépense de talent. Vallès, qui prédit la mort de la "chronique-commère», spécialité du «journalisme causotier qui a la frivolité pour muse », s'indigne : «Dieu sait (...) si l'on a dépensé, à cette besogne stérile, du talent!». L'inanité de ce journalisme d'actualité occupé à peindre, non pas « la vie courante ", mais la « vie factice ", voue les pages écrites - et leurs rédacteurs - à un oubli certain : « on ne s'occupera pas plus [des chroniqueurs] que s'ils n'avaient jamais écrit, et ils auront fait un métier de dupes !»; bien plus, « (...) un passé de chroniqueur sera, pour un écrivain, non pas un marchepied, mais un boulet. Le public ne voudra pas croire que cet enfileur de babioles puisse écrire jamais une comédie, un roman ou un drame, dans lesquels il faudra noter non pas les cancans du jour, mais les cris d'une époque en travail et d'un monde en marche.» (Vallès, 1867) Défaut de crédibilité, mais aussi peut-être même perte de facultés: Barbey d'Aurevilly prévient les jeunes chroniqueurs que «lorsqu'on a couru le petit fait pendant quelque temps, on n'est plus capable que de courir le petit fait. L'idée méprisée devient infidèle. L'invention ne vous prend plus dans ses bras féconds. » (Barbey, 1883 : 83) 


\section{Plaidoyers pour la chronique}

17 Dans les défenses de la chronique se mêlent les concessions et les véritables plaidoyers en faveur du genre. A un moindre niveau, on reconnaît ainsi au chroniqueur des qualités spéciales. Maupassant, qui opère une nette distinction entre chroniqueur et romancier, relève un talent propre aux « vrais chroniqueurs », qui seraient « tout aussi rares et aussi précieux que les vrais romanciers »:

Il faut que [le chroniqueur] soit sans cesse le favori des lecteurs, qu'il s'efforce sans cesse de les séduire ou de les convaincre. Il a besoin pour cet effort constant, d'une incroyable énergie, d'un tempérament infatigable, d'un esprit et d'une présence d'esprit sans limites. Le mépris systématique des romanciers pour leurs frères $\mathrm{du}$ journalisme n'empêchera point qu'il soit aussi difficile au directeur d'un grand journal de découvrir un chroniqueur, qu'il est difficile à un éditeur de mettre la main sur un auteur. (Maupassant, 1884)

$18 \mathrm{Si}$, selon Jules Lemaitre, « (...) écrire des chroniques dans un journal est une des besognes les plus vaines auxquelles un homme puisse consacrer ses jours périssables », il extrait cependant de la masse des improvisations quotidiennes des chroniqueurs au style marquant (Lemaitre, 1887 : 265-269).

19 Nombreux aussi sont les véritables plaidoyers pour le genre qui relèvent la littérarité et les hautes ambitions de la chronique. P.-J. Stahl demande un "examen sérieux de la critique" pour le recueil des chroniques de Villemot au Figaro (Villemot, 1858: XXIV). Dans le Gil-Blas, Nestor (Henry Fouquier) répond à Maupassant, et réfute l'idée selon laquelle le chroniqueur n'aurait pas besoin d'idées générales :

Je suis assez sincèrement modeste quand il s'agit des chroniqueurs, et en particulier de moi, pour parler avec quelque fierté et quelque orgueil de la chronique ! Bien ou mal, avec plus ou moins de tour de main, loin d'être de simples amuseurs, nous sommes des semeurs d'idées. Les chroniques publiées depuis vingt ans, réunies en volumes, donneraient une encyclopédie de notre temps. Sous une forme tantôt légère, tantôt plus sévère, et qui importe peu, la chronique a tout abordé, religion, politique, critique, histoire, morale. Un jour c'est une page des Essais de Montaigne qui s'égare dans un journal; un autre jour, une joyeuse moralité de Rabelais; c'est une page d'un pamphlet de Voltaire; c'est un chapitre de Beccaria ou une diatribe de Desmoulins. Vous pensez bien que je ne veux pas dire que nous soyons des Voltaires et des Montaignes! La sottise serait trop forte. Mais, chacun à son rang, nous continuons la grande œuvre littéraire, et cette œuvre, même chez ceux qui s'en défendent, c'est de répandre, de prêcher, d'imposer au monde des idées générales! (Nestor, 1884)

Ce rôle de moralistes modernes est une fonction régulièrement revendiquée par les chroniqueurs; Vapereau lui-même précise au sujet des boulevardiers que «les plus forts d'entre les historiens de ces misères élégantes ne s'y enferment pas ». Au milieu de leur bavardage périodique et des cancans, «ils savent jeter (...) quelque fine satire, une observation philosophique sous une apparence superficielle; ils se vengent par l'ironie railleuse de la nécessité acceptée d'assaisonner chaque jour pour le public, un menu de sottises. » (Vapereau, $1867: 299$ )

Conscient de vivre à un "moment curieux », Emile Zola estime, en 1865, que son époque «a besoin de moralistes, de chroniqueurs qui marquent les phases de sa maladie, ses fièvres et ses palpitations » (Zola, 2002: 675). Les introductions des recueils de chroniques donnent rétrospectivement cette valeur aux articles qui les composent. Dans l'« Avant- 
propos » de son recueil intitulé La Sagesse parisienne (1885), qui réunit des articles parus dans le Gil-Blas, Henry Fouquier souligne que «[la pente naturelle de son esprit l'a souvent conduit à chercher, dans les incidents même les plus frivoles, les conseils, les avertissements, les espérances ou les menaces qui sortent des petits faits de la vie contemporaine ». Jules Claretie précise ses intentions de chroniqueur, dans la préface de la troisième année de son recueil La Vie à Paris :

Je m'efforce, en effet, dans ces causeries, comme je voudrais le faire dans mes romans, de fixer l'accent, l'allure, les particularités de cette vie moderne si compliquée et si attirante. Nous vivons dans un temps bizarre, mais très propice aux observateurs. Plus ce monde est fou, plus il est curieux. Le romancier, qui l'étudie, peut y faire plus facilement œuvre de peintre, et le journaliste office de moraliste.

Moraliste ! voilà ( $\mathrm{V}$ ?) un bien gros mot pour des causeries à la plume ! C'est pourtant un titre pareil que je voudrais revendiquer. (Claretie, 1882 : I-II)

Essai ou encyclopédie donc, mais aussi pamphlet. La chronique a pu être un "contrepouvoir » et un espace de « résistance par l'ironie à l'Empire » (Thérenty, 2011 : 965), dans les dernières années du régime, en particulier, avec les mesures de libéralisation de la presse de 1868. Retraçant les évolutions de la chronique, Jules Claretie rappelle que «la chronique qui parlait la langue de Candide avec Valentin de Quévilly 'le bon jeune homme', allait, un jour, emprunter le style de Duvert et Lauzanne et, se faisant pamphlet, contribuer à la chute d'un empire. » La superficialité cache un potentiel de subversion: " cette 'commère' est une force et son petit air de fifre a sa valeur dans la mêlée. C'est le petit turlututu dont Frédérick II parlait un soir de bataille: ' Messieurs, le héros de la journée, c'est un musicien de quinze ans qui, sous les balles, n'a cessé de jouer de son turlututu'.» (Claretie, $1880: 3$ )

Chargé des «Causeries» du journal républicain La Tribune en 1868-1869, Zola mobilise une grande variété de dispositifs textuels (récits, lettres, saynètes, dialogues, allégories, voire attaques directes contre le régime impérial à l'approche des élections législatives de 1869), variété qu'autorise le cadre souple de la chronique, pour livrer une relecture satirique de l'histoire du second Empire (Carvalhosa, 2013). Rochefort emblématise cette forme d'écriture oblique, dont «l'ironie est (...) [l'] arme de prédilection, (...) elle touche toujours juste, et blesse souvent jusqu'au sang ». La mise en recueil, avec les seuils stratégiques que sont le titre et la préface, explicite la signification générale de ses articles :

(...) M. Rochefort vient de publier une seconde série des Français de la décadence, sous le titre particulier de la Grande bohême. C'est encore, sous la forme morcelée d'articles d'actualité, l'histoire plus ou moins satirique de l'année écoulée. Le ton en est toujours aussi ferme et aussi vif, et la pensée côtoie, sans la franchir, la limite tracée par l'administration devant les hardiesses du petit journalisme. Mais ce que cinquante articles de journal ne disent pas, la préface le dit en quatre pages. L'ironie si mordante de M. Prévost-Paradol est dépassée. Il est impossible de pousser la satire plus loin, en donnant ses raisons de s'en abstenir. Le plan de comédie politique qu'il esquisse, avec ses allusions transparentes, montre jusqu'à quel point l'audace peut être sauvée par l'esprit. (Vapereau, $1867: 301$ )

La question même de la péremption de la chronique - argument fort contre le genre doit être reconsidérée, en envisageant plusieurs lectures possibles de la chronique, à court et à long terme. Jules Lemaitre voit ainsi une possibilité, pour la chronique, de survivre sur le long terme : 
La chronique sera donc, si vous voulez, de la poussière de littérature ; mais c'est de la littérature encore.

Et c'est aussi ou ce peut être de la poussière d'histoire. Si vous relisez les chroniques du mois dernier, il est probable qu'elles vous sembleront insipides, superflues, et que vous n'y apprendrez rien. Mais lisez, pour voir, des recueils de chroniques d'il y a vingt ans. Là encore vous trouverez sans doute beaucoup de fatras et un vide lamentable; mais parfois, noyé dans cette insignifiance, un détail vous frappera, un détail caractéristique d'une époque et dont l'écrivain n'avait peut-être pas soupçonné la valeur future. Les chroniques des journaux, en vieillissant, deviennent mémoires.

Celles d'aujourd'hui paraîtront prodigieusement intéressantes dans cent ans. Seulement il y en aura trop. (Lemaitre, $1887: 268$ )

Il s'agirait alors de distinguer au moins deux temporalités (et donc deux modes) de lecture possibles pour la chronique, selon la distance temporelle qui s'est établie entre son écriture et sa lecture : une lecture contemporaine (ou presque) de l'écriture (c'est le cas de la chronique qui paraît dans le journal), attachée aux grâces du style et/ou au contenu ; une lecture différée, " historique ", de la chronique, qui la considère comme un document, des «mémoires». Le propos de Lemaitre n'est pas original : c'est l'ambition affichée de Jules Claretie avec la publication de ses recueils de chroniques du Temps, La Vie à Paris, que de laisser aux générations futures la petite histoire de son époque, qui complète la grande. Sans nier la péremption programmée de ses articles (Claretie, 1882: VI), et mettant « la chronique à son rang dans l'échelle littéraire - ni trop haut, ni trop bas ", il espère que

(...) ces Mémoires des Années Parisiennes formeront un ouvrage de chercheurs, de fureteurs, un livre de coin de bibliothèque, qu'on sera très aise de trouver, comme on est enchanté de rencontrer, sur les quais, une gravure de Debucourt, lorsqu'on voudra savoir un peu comment vivaient les Français de ce temps, qui est bien, soit dit entre nous, le plus bizarre et le plus affolé qui ait jamais marqué sur un baromètre moral. (Claretie, 1880 : IX)

Vocation d'autant plus légitime que Claretie considère qu'« aujourd'hui d'ailleurs, plus que jamais, les historiettes de la chronique se rapprochent de l'histoire. Elle écrit sur la marge du grand livre officiel; elle met des notes au bas des pages, elle complète le chapitre. Elle vit de faits et de vérités. " (Claretie, 1880:3) Le Grand Dictionnaire universel $d u X^{X} X^{e}$ de Larousse considère lui aussi - de manière plus ambiguë, car il est aussi un contempteur de la «chronique-commère» - que les chroniques, dans leurs insignifiances mêmes, seront des documents pour le futur :

Les chroniqueurs sont les photographes reproduisant par tous les temps la société prise sur le fait. Ils amusent, à l'heure où ils écrivent, un grand nombre de lecteurs passionnés pour leurs cancans de chaque jour ; mais ils amuseront bien plus encore les curieux de l'avenir, qui, sous l'originalité de ces indiscrétions rapides, retrouveront la physionomie intime d'une époque disparue.

L'analyse omet au moins un cas de figure : la possibilité, pour la chronique, de survivre par ses seules qualités littéraires. Ce sont sans doute les chroniques répondant le moins à l'impératif de l'actualité (qui s'en échappent par la fictionnalisation et/ou par un travail esthétique de la matière) qui supportent le mieux ce type de lecture.

Dans le dernier quart du siècle pointe déjà une nouvelle forme de plaidoyer pour la chronique. Avec l'émergence, dans le journalisme français, du primat (encore relatif) de l'information, emblématisé par le reportage, la chronique apparait comme un genre menacé $^{10}$. Aurélien Scholl considère qu' «avec Villemessant vont disparaître tout doucement les chroniques et les nouvelles à la main. Depuis quelque temps déjà, on 
entendait cette plainte des éditeurs de journaux : Il n'y a plus de chroniqueurs!», ce qui s'explique par les nouvelles attentes du public: «Il n'y a plus de chroniqueurs, parce qu'on n'en a plus besoin. L'attention des lecteurs, le goût du public se sont déplacés. On est fatigué de sornettes ; on demande un ragoût plus substantiel. » (Scholl, 1882 : 256-257) A l'occasion de la mort de Jules Noriac, en 1882, Jules Claretie fait un bilan nostalgique de la période boulevardière qui appartient à un temps révolu - et glorieux - du journalisme ("les journalistes littéraires d'autrefois»), même si quelques "maréchaux de la chronique » tiennent encore le haut de page :

Ce qui est certain, c'est qu'il représentera longtemps tout un genre spécial de la littérature parisienne, un genre particulier, où il fallait non seulement des renseignements et des faits comme aujourd'hui, mais de l'humour, de l'observation, la rapidité dans l'esprit, la clarté dans le style, du mordant et de la délicatesse à la fois... L'érudition même ne déplaisait point quand Monselet s'en mêlait. Léo Lespès, qui fut populaire un moment, Auguste Villemot, le fin bourgeois de Paris, Noriac, que la femme d'esprit qui signait Horace de Lagardie eut la sévérité de railler, Albert Wolff, Aurélien Scholl, Henri Rochefort, les satiriques toujours en verve après vingt-cinq ans, formaient ce groupe choisi qui a sa place et qui aura peut-être son histoire.

On les a remplacés par des reporters.

Leurs successeurs croient sans doute écrire des pages.

Lorsque Jules Noriac réunissait ses vieux articles, il leur donnait simplement ce titre : Folioles.

Il y a de ces folioles qui n'ont point jauni et qui dureront. (Claretie, $1882: 429-430)$

La chronique du XIX ${ }^{e}$ siècle se présente donc à nous, "curieux de l'avenir ", comme un genre protéiforme. Ecriture souple, et modelée par l'individualité/ le style du causeur, la causerie participe du décloisonnement de la chronique - même si la chronique-causerie, devenue causerie chronique, a pu s'enfermer dans l'imitation de modèles à succès (le Courrier de Paris ou la chronique populaire). Les frontières de la chronique avec la littérature ont été également objet de litige : le passage du journal au livre, et la littérarité de la chronique, en particulier, ont été au cœur de débats passionnés. Les hautes ambitions nourries par certains chroniqueurs pour le genre semblent néanmoins réalisées: les recherches contemporaines sur la presse $d u x x^{e}$ siècle redécouvrent ce massif impressionnant, et la forme littéraire éminemment créatrice que fut, sous certaines plumes, la chronique.

\section{BIBLIOGRAPHIE}

(Les références entre parenthèses pour La Vie à Paris de J. Claretie et L'année littéraire de G. Vapereau renvoient à l'année considérée par chaque ouvrage)

BARBEY D'AUREVILLY, Jules (1883). Les ridicules du temps, Paris : E. Rouveyre et G. Blond (article paru le 24 février 1866 dans Le Nain Jaune). 
CARVALHOSA, Sandrine (2013). « Zola et la causerie. Un apprentissage de l'écriture oblique (1865-1870) », in Les Cahiers naturalistes, n. 87, pp. 43-62.

CHABRIER, Amélie (2013). « Les chroniques de Timothée Trimm dans Le Petit Journal : une défense et illustration de la chronique populaire ", in Matière et esprit du journal, du Mercure Galant à Twitter , Alexis Lévrier et Adeline Wrona (orgs.), Paris : Presses de l'université Paris-Sorbonne, pp. 181-193.

CLARETIE, Jules (1880). La vie à Paris : 1880-1885. Année 1, Paris : V. Havard, 1881-1886.

CLARETIE, Jules (1882). La vie à Paris : 1880-1885. Année 3, Paris : V. Havard, 1881-1886.

DIAZ, José-Luis (2011). « Avatars journalistiques de l'éloquence privée », La Civilisation du journal, D. Kalifa, P. Régnier, M.-È. Thérenty, A. Vaillant (orgs.), Paris : Nouveau Monde Editions, « Opus magnum », pp. 691-715.

LAROUSSE, Pierre (1866-1877). Articles « Causerie », "Chronique », « Chroniqueur », Grand Dictionnaire universel du XIX ${ }^{e}$ siècle, Paris : Administration du Grand Dictionnaire universel. LEMAîTRE, Jules (1887). Les contemporains : études et portraits littéraires. $3^{e}$ série, Paris : H. Lecène et H. Oudin.

MAUPASSANT, Guy de (1884). « Messieurs de la chronique », Gil-Blas, 11 novembre 1884.

NESTOR, (Henry FOUQUIER) (1884). « Chroniqueurs et romanciers », Gil-Blas, 12 novembre 1884.

SARCEY, Francisque (1869a). « Les conférences », conférence au boulevard des Capucines, Revue des cours littéraires de la France et de l'étranger, Paris : Germer Baillière, pp. 19-24.

SARCEY, Francisque (1869b). « Le Journaliste », conférence au Boulevard des Capucines, Revue des cours littéraires de la France et de l'étranger, Paris : Germer Baillière, pp. 331-336.

SCHOLL, Aurélien (1882). Mémoires du trottoir, Paris : E. Dentu.

THÉRENTY, Marie-Ève (2007). La littérature au quotidien. Poétiques journalistiques au XIXe siècle, Paris : Editions du Seuil, coll. « Poétique ».

THÉRENTY, Marie-Ève (2011). « La chronique », La Civilisation du journal, D. Kalifa, P. Régnier, M.-È. Thérenty, A. Vaillant (dir.), Paris : Nouveau Monde Editions, « Opus magnum », pp. 953-968.

VALLÈs, Jules (1866). « Timothée Trimm et Thérésa », Figaro, 21 janvier 1866.

VALLÈs, Jules (1867). « Hier-Demain », Le Nain Jaune, 14 février 1867.

VAPEREAU, Gustave (1866). L'année littéraire et dramatique. 1866, Paris : L. Hachette, 1858-1868.

VAPEREAU, Gustave (1867). L'année littéraire et dramatique. 1867, Paris : L. Hachette, 1858-1868.

VAPEREAU, Gustave (1876). Dictionnaire universel des littératures, Paris : Hachette.

VILLEMOT, Auguste (1858). La vie à Paris : chroniques du Figaro. Précédées d'une Étude sur l'esprit en France à notre époque. Série 1, Paris : Hetzel, M. Lévy frères.

WOGAN, Émile Tanneguy de (1899). Manuel des gens de lettres : le journal, le livre, le théâtre, Paris : Firmin-Didot.

zolA, Émile (2002). « Lettres d'un curieux », Euvres complètes, H. Mitterand (éd.), T. I, Paris : Nouveau Monde. 


\section{NOTES}

1. Jules Claretie s'en fait encore l'écho en 1882 : «J'aurais presque envie de dire que le journal, qui donne à tout le monde à la fois le même sujet de conversation banale, a tué la causerie et la conversation, si l'on ne répétait pas depuis plus de cent ans que la conversation est morte.» (Claretie, $1881: 375$ )

2. Du début de l'« ère médiatique » aux débuts du journalisme d'information en France, bien qu'il faille plutôt parler dans le second cas d'une évolution lente, commencée avant 1885 ; c'est le corpus considéré ici qui impose cette borne finale.

3. La référence au vicomte de Launay fonctionne comme un véritable cliché, souligné comme tel par les chroniqueurs : «A propos de chronique et de chroniqueurs, voici un nouveau venu dans la carrière, un transfuge du feuilleton des théâtres, M. Jules de Prémaray. (...) Il commence, comme tout le monde a commencé, par l'hymne de rigueur en l'honneur du vicomte de Launay. C'est le musa, mihi causas memora, en d'autres termes l'invocation à la muse, ou, plus chrétiennement, la prière à Notre-Dame-de-Bon-Secours des feuilletons dans l'embarras (...) ». («Courrier de Paris », Figaro, 31 janvier 1858)

4. La causerie de Trimm occupe la place dévolue au Premier-Paris, dans les journaux politiques (première page). L'expression, moqueuse, est présente dans le Figaro.

5. Voir aussi le « Manuel du Courriériste » de J. Noriac, publié pour la première fois dans le Figaro, le 26 juillet 1857 et repris dans son recueil intitulé Le 101e régiment, qui livre la recette d'un Courrier de Paris.

6. On appréciera le «compliment» de B. Jouvin à Timothée Trimm (Figaro, 5 octobre 1865) : «Timothée Trimm est tiré chaque soir à plus de deux cent mille exemplaires : cela suppose, au bas prix, un million de lecteurs, - également au bas prix. Mais enfin un meunier, quand il a tiré $\mathrm{du}$ grain du froment la fine fleur de la farine, ne jette pas le son dans l'eau de son moulin. Un écrivain, dans l'impuissance de fonder sa réputation sur la "qualité » n'est point absolument méprisable en demandant la vogue à la « quantité » de ses lecteurs. Ne pouvant être farine, mieux vaut encore être son que de n'être rien!»

7. Barbey d'Aurevilly s'insurge contre les journaux à un sou « qu'on [achète] avec furie, et non pas simplement les cochers et les femmes de chambre, mais les maîtres... devenus les rivaux de la lecture de leurs femmes de chambre et de leurs cochers. » (Barbey d'Aurevilly, 1883: 76)

8. Figaro, 5 octobre 1865.

9. Le chroniqueur commet régulièrement des erreurs. Voir par exemple Figaro, 5 janvier 1865 ou 22 janvier 1865.

10. La Revue Illustrée, "Critique littéraire ", 5 septembre 1906, évoque également le cas de la critique: «Depuis que, sacrifiant à la déesse Information, les causeries qui firent le régal des lettrés d'autrefois au point que leurs signataires déplaçaient avec eux la clientèle d'un journal, les grands quotidiens ont réduit de plus de moitié la place d'ordinaire réservée aux maîtres de la chronique pour en céder une large part aux princes du reportage, la critique littéraire et la critique d'Art furent marquées en tête des listes de proscription. » 


\section{RÉSUMÉS}

Nombre de formules (et de titres) au XIX e siècle assimilent le journal à un « causeur gigantesque » (Claretie), à une « conversation écrite ». La chronique journalistique est le lieu privilégié de cette causerie périodique. Lieu emblématique des contraintes législatives qui s'exercent sur la presse au XIX ${ }^{\mathrm{e}}$ siècle, la chronique est aussi, grâce à sa plasticité formelle, un espace (paradoxal) de liberté. Nous chercherons à décrire quelques formes prises par cette greffe de la causerie familière dans la chronique, et à cerner les enjeux qui lui sont attachés. Considérée comme un bavardage inconsistant et/ou comme les mémoires du temps, où « les curieux de l'avenir [...] retrouveront la physionomie intime d'une époque disparue», décriée pour ses facilités et regrettée avec l'avènement $\mathrm{du}$ reportage, la chronique-causerie nous apparaît aujourd'hui comme une forme littéraire éminemment créatrice.

$\mathrm{XIX}^{\text {th }}$ century newspapers have been described as "written conversation". Newspaper columns are the most prominent location of this talk. They were a target of legislative constraints, that were exerted on the press at the time, but they were also a space of freedom. We will endeavour to describe the modalities of this transfer of speech into writing. We will also try to identify the issues at stake. They were considered as inconsistent chatter, and/or as annals in which "future generations [...] will be able to rediscover the intimate character of a bygone era"; they were missed after the advent of the more factually orientated news reports. It appears to us today as a highly creative literary format.

\section{INDEX}

Mots-clés : chronique, causerie, journal, frontières, littérature

Keywords : newspaper columns, talk, press, frontiers, literature

\section{AUTEUR}

\section{SANDRINE CARVALHOSA}

Montpellier III

sandrine.carvalhosa-martins[at]univ-montp3.fr 